\title{
Science and fashion: inflammation and oxidative stress in psychiatry
}

\author{
C. Arango ${ }^{1}$
}

Published online: 23 January 2018

c) Springer-Verlag GmbH Germany, part of Springer Nature 2018

Science is not immune to fashion, and from time to time, some topics become very trendy in different areas of medicine. The booming interest in inflammation and oxidative stress in medical research in the last decade is a good example. Areas such as oncology and cardiology have taken the lead regarding interest in the role of inflammatory and oxidative stress markers as signs of underlying pathophysiology and their potential therapeutic implications. Psychiatry has followed that lead, and the number of papers addressing immune/inflammatory and oxidative/nitrosative markers has burgeoned in recent years. Many studies show a relationship between these markers, in the form of an imbalance between pro- and anti-inflammatory activity, and almost any psychiatric disorder [1-4]. Two papers in this issue deal with oxidative disorders in different psychiatric disorders.

Bartoli et al. [5] provide a systematic review and metaanalysis of papers assessing uric acid levels in patients with major depressive disorder (MDD). Uric acid is a major antioxidant that accounts for high free radical scavenging activity in humans, including in the central nervous system. In the meta-analysis, MDD patients had significantly lower uric acid levels than healthy controls. Most importantly, the difference with healthy controls was significant only among studies with drug-naïve patients, but not among those involving treated patients. In a limited number of longitudinal studies ( $n=4$ with 220 patients) assessing uric acid, levels significantly increased after antidepressant treatment. Of course, the results raised a very relevant hypothesis to be tested regarding the effects of drugs used to treat depression on the oxidative and inflammatory systems. However, the present study cannot rule out the possibility that the change in the studies' antioxidants is not related to another

C. Arango

carango@hggm.es

1 Child and Adolescent Psychiatry Department, Hospital General Universitario Gregorio Marañón, School of Medicine, Universidad Complutense, IiSGM, CIBERSAM, Madrid, Spain epiphenomenon (such as a result of the improvement or stress reduction).

Jordan et al. [6] precisely address one of the most fundamental questions on the relationship between oxidative stress and psychiatric disorders (in their study, schizophrenia and depression), that is, whether oxidative stress factors and immune dysregulation are related to the pathophysiology of those disorders or are just a mere epiphenomenon. In a longitudinal study with drug-naïve first-episode patients, they show that most elevated markers in patients were not related to age, sex, disease severity, medication, or adipose tissue mass. However, markers such as 8 -iso-PGF2 $\alpha$ were associated with smoking or endocrine stress axis activation. The authors highlight the role of lipid peroxidation and the importance of taking into account other confounding factors in biomarker studies of oxidative stress. Despite the fact that, at baseline or after 6 weeks of treatment, oxidative stress measures did not seem to be affected by age, gender, or occasional cannabis use in the whole group of subjects or in each diagnostic group separately, the results should be considered preliminary, given the small sample size in each of the study groups. It is noteworthy that, in schizophrenia patients, urinary marker 8 -iso-PGF2 $\alpha$, already elevated at baseline, was further increased after 6 weeks of treatment, suggesting possible cumulative damage to membrane lipids. As the authors state, the major source of 8 -iso-PGF2 $\alpha$ in healthy humans seems to be free radical-catalysed lipid peroxidation. Meanwhile, in pathological conditions, enzymatic over-expression due to inflammation and activation of COX-2 is another source of 8 -iso-PGF $2 \alpha$ production. Therapeutic implications derived from this study are that mitigation of the lipid peroxidation could be addressed with the use of omega-3 fatty acids, eicosapentaenoic acid, or $\mathrm{N}$-acetyl cysteine (NAC). In a recent RCT, administration of NAC versus placebo in first-episode psychosis patients improved neurocognition. Interestingly, NAC also improved positive symptoms in the subgroup of patients with higher glutathione peroxidase activity [7].

It is relevant to mention the lack of specificity of the above findings, with reports of inflammatory and oxidative 
stress marker abnormalities in almost all psychiatric (and neurological) disorders so far studied. However, as inflammatory and oxidative stress markers may predict clinical, cognitive, and neurobiological outcomes [8], there is a clear need to identify biomarkers in first-episode patients with different psychiatric disorders. This strategy may help to group patients who may respond to different treatment strategies. This is regardless of whether oxidative and inflammatory markers are intrinsically part of the pathophysiology of psychiatric disorders, as genetic studies repeatedly point out, or they are a consequence of the disorders or associated features (e.g. psychological stress, nutrition, medication, comorbid illnesses, etc.). There are also some preliminary studies showing that biomarkers related to immunoinflammatory or oxidative stress abnormalities have been associated with treatment response [9]. Taken together, these make immunological oxidative stress a fashion that may ultimately improve the course in subgroups of patients with different psychiatric disorders.

\section{References}

1. Friedrich MJ (2014) Research on psychiatric disorders targets inflammation. JAMA 312:474-476

2. Leza JC, García-Bueno B, Bioque M, Arango C, Parellada M, Do K, O’Donnell P, Bernardo M (2015) Inflammation in schizophrenia: a question of balance. Neurosci Biobehav Rev 55:612-626
3. Soria V, Uribe J, Salvat-Pujol N, Palao D, Menchón JM, Labad $\mathbf{J}$ (in press) Psychoneuroimmunology of mental disorders. Rev Psiquiatr Salud Ment https://doi.org/10.1016/j.rpsm.2017.07.006

4. Pariante CM (2017) Why are depressed patients inflamed? A reflection on 20 years of research on depression, glucocorticoid resistance and inflammation. Eur Neuropsychopharmacol 27:554-559

5. Bartoli F et al (2018) Antioxidant uric acid in treated and untreated subjects with major depressive disorder: a meta-analysis and meta-regression. Eur Arch Psychiatry Clin Neurosci 268:107118. https://doi.org/10.1007/s00406-017-0817-7 (this issue)

6. Jordan W et al (2018) Oxidative stress in drug-naïve first episode patients with schizophrenia and major depression: effects of disease acuity and potential confounders. Eur Arch Psychiatry Clin Neurosci 268:129-144. https://doi.org/10.1007/s00406-016-07497 (this issue)

7. Conus P, Seidman LJ, Fournier M, Xin L, Cleusix M, Baumann PS, Ferrari C, Cousins A, Alameda L, Gholam-Rezaee M, Golay P, Jenni R, Wool TUW, Keshavan MS, Eap CB, Wojcik J, Cuenod M, Buclin T, Gruetter R, Do KQ (in press) $N$-acetylcysteine in a double-blind randomized placebo-controlled trial: toward biomarker-guided treatment in early psychosis. Schizophr Bull https://doi.org/10.1093/schbul/sbx093

8. Fraguas D, Díaz-Caneja CM, Rodríguez-Quiroga A, Arango C (2017) Oxidative stress and inflammation in early onset first episode psychosis: a systematic review and meta-analysis. Int $\mathbf{J}$ Neuropsychopharmacol 20:435-444

9. Fond G, d'Albis MA, Jamain S, Tamouza R, Arango C, Fleischhacker WW, Glenthøj B, Leweke M, Lewis S, McGuire P, Meyer-Lindenberg A, Sommer IE, Winter-van Rossum I, Kapur S, Kahn RS, Rujescu D, Leboyer M (2015) The promise of biological markers for treatment response in first-episode psychosis: a systematic review. Schizophr Bull 41:559- 73 